\title{
Hypermethylation of TMEM240 Involved in Expression Deficiency Predicts Poor Hormone Therapy Response and Disease Progression in Breast Cancer
}

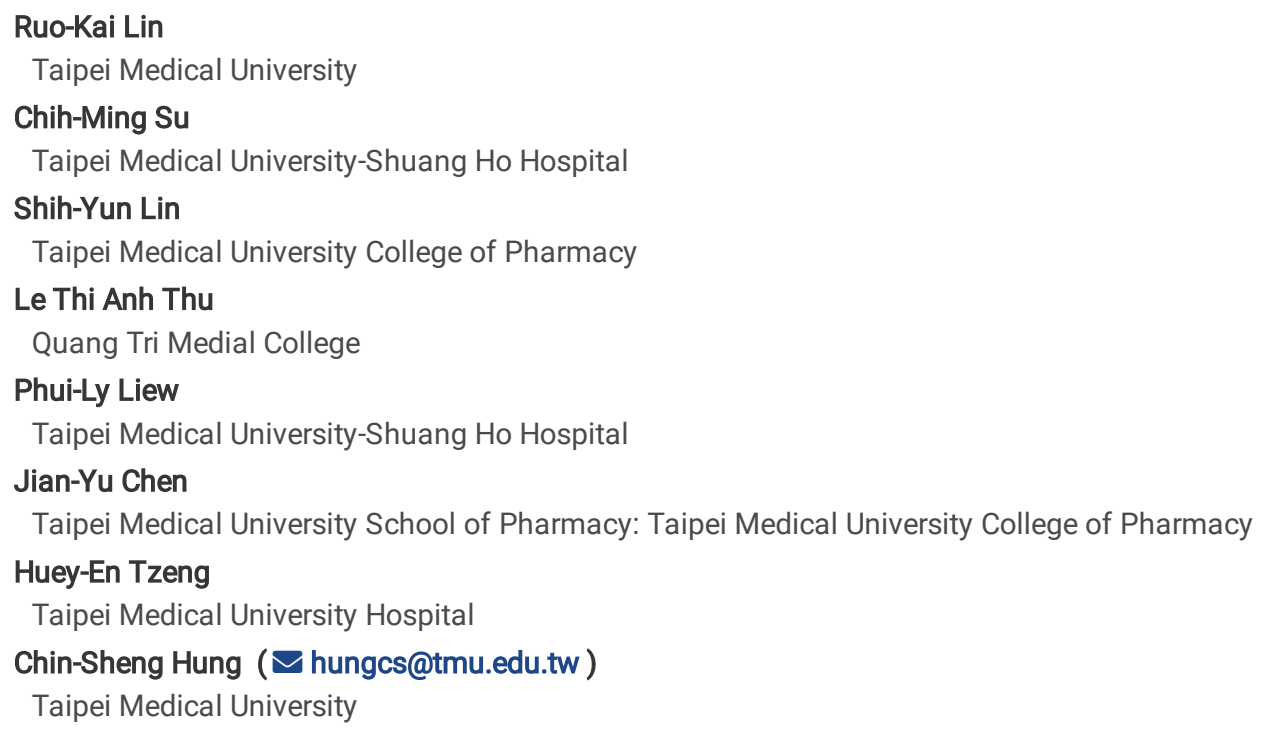

Keywords: TMEM240, breast cancer, DNA methylation, circulating cell-free DNA, disease progression, tumor suppressor genes, proliferation, cell motility and hormone therapy

Posted Date: November 10th, 2021

DOI: https://doi.org/10.21203/rs.3.rs-1021973/v1

License: (c) (i) This work is licensed under a Creative Commons Attribution 4.0 International License. Read Full License 


\section{Abstract}

\section{Background:}

Approximately $25 \%$ of patients with early-stage breast cancer experience cancer progression throughout the disease course. Alterations in TMEM240 in breast cancer were identified and investigated to monitor treatment response and disease progression.

\section{Methods:}

Circulating methylated TMEM240 in the plasma of breast cancer patients was used to monitor treatment response and relapse events. Illumina methylation arrays were used to identify novel hypermethylated CpG sites and genes related to poor hormone therapy response. Quantitative methylation-specific real-time polymerase chain reaction (QMSP), quantitative real-time reverse transcription PCR, and immunohistochemical analyses were performed to measure DNA methylation, mRNA and protein expression levels in 335 breast samples from Taiwanese and Korean patients. Kaplan-Meier curves, Cox proportional hazards regression and receiver operating characteristic curves were used to analyze 10-year survival and disease progression. The Cancer Genome Atlas (TCGA) dataset was used to investigate TMEM240 alterations in Western countries. Transient transfection and knockdown of TMEM240 were performed to determine its biological functions and its relationship to hormone drug treatment response in breast cancer cells.

Results: Aberrant methylated TMEM240 was identified in breast cancer patients with poor hormone therapy response using genome-wide methylation analysis in the Taiwan and TCGA breast cancer cohorts. A cell model showed that TMEM240, which is localized to the cell membrane and cytoplasm, represses breast cancer cell proliferation and cell migration. TMEM240 protein expression was observed in normal breast tissues, but not detected in $88.2 \%(67 / 76)$ of breast tumors and in $90.0 \%(9 / 10)$ of metastatic tumors from breast cancer patients. Almost all triple-negative breast cancer patients $(95.7 \%, 22 / 23)$ had deficient TMEM240 protein expression. QMSP revealed that in 54.5\% (55/101) of Taiwanese breast cancer patients, the methylation level of TMEM240 was at least 2-fold higher in tumor tissues than in the matched normal breast tissues. Patients with hypermethylation of TMEM240 had poor 10-year overall survival $(p=0.003)$ and poor treatment response, especially hormone therapy response $(p<0.001)$. Prediction of disease progression based on circulating methylated TMEM 240 was found to have $87.5 \%$ sensitivity, $93.1 \%$ specificity, and $90.2 \%$ accuracy, better than the currently used biomarkers CEA and CA-153.

Conclusions: Circulating methylated TMEM240 is a potential biomarker for treatment response and disease progression monitoring in breast cancer.

\section{Background}

Breast cancer is the most common cancer in women, surpassing lung cancer, worldwide [1-3]. The estrogen-dependent nature of breast cancer is the fundamental basis for hormone therapy. The hormone receptor-positive human epidermal growth factor receptor 2-negative (HR+/HER2-) subtype, which is characterized by the expression of estrogen receptor (ER) and/or progesterone receptor (PR) without HER2 overexpression/amplification, accounts for approximately $70 \%$ of breast cancer patients. However, estrogen-independent growth often exists de novo at diagnosis or develops during the course of hormone therapy. Nearly $20 \%-30 \%$ of patients with early-stage disease become metastatic throughout the disease course [4]. Therefore, ER expression alone is insufficient in predicting endocrine therapy efficacy [5]. A significant number of these patients will develop either primary or secondary hormone resistance, prompting the need for tests that can predict treatment response before treatment options are chosen. The initiation and progression of cancer, which is conventionally considered a genetic disease, involve epigenetic abnormalities [6]. Genomic screening of 98 different primary human tumors revealed that on average, approximately 600 aberrantly methylated CpG islands exist in each tumor [7]. Combined analysis of data from Taiwanese individuals for whom both data on breast cancer tissue and data on clinical treatment response are available in the TCGA database has shown that hypermethylation of the gene encoding transmembrane protein gene 240 (TMEM240) is a biomarker of poor hormone therapy response in breast cancer. TMEM240 encodes a transmembrane domain-containing protein found in the brain and cerebellum. In studies of patients from France, Germany, the Netherlands, Colombia, Japan, and China, mutations in TMEM240 have been found to cause spinocerebellar ataxia 21 (SCA21) with mental retardation, severe cognitive impairment, and hypokinetic and hyperkinetic movement disorders [8-11]. The pathogenic mechanism of SCA21 may be mediated through the induction of early gliosis and lysosomal impairment by mutant TMEM240 [12]. Hypermethylation of TMEM240 has been found in colorectal cancer (CRC) [13, 14]. Few reports are available regarding TMEM240 in women with cancer, and the role of TMEM240 in breast cancer remains unclear.

Advances in detection technology have reduced breast cancer death rates in several Western countries [15]. Therefore, developing biomarkers for treatment response can improve patient outcomes. For current disease progression monitoring for breast cancer patients, simultaneous use of the two serum markers CA-153 and carcinoembryonic antigen (CEA) shows that the early diagnosis of metastasis in up to $60 \%-80 \%$ of patients with breast cancer is not sensitive enough to monitor disease progression in real time [16-18]. Therefore, no dynamic monitoring system for accurately and sensitively measuring recurrence or metastasis events is available in current clinical practice. Circulating cell-free DNA (ccfDNA) in plasma can be used for the noninvasive sampling of cancer cells obtained from patients with breast cancer [19]. Cells release cell-free DNA through a combination of apoptosis, necrosis, and active secretion. Cancer cells, as well as cells in the tumor microenvironment, can produce ccfDNA. Multiple genetic and epigenetic alterations are found in ccfDNA [20]. Assays of circulating methylated DNA (cmDNA) could be used for outcome prediction in metastatic breast cancer patients treated with chemotherapy and/or multikinase inhibitors [21]. Circulating methylated TMEM240 can be

Page $2 / 21$ 
successfully detected in patients with CRC [13]. In this study, the promoter methylation level, the expression level and the biological functions of TMEM240 will be clarified. Whether circulating methylated TMEM240 can be detected in blood from individuals with breast cancer and its association with treatment response and disease progression will also be investigated.

\section{Methods}

\section{Patients and tissue, plasma collection}

A total of 335 Taiwanese clinical samples, including 137 human breast tumor tissues, 137 adjacent normal breast tissue samples and 61 plasma samples, were obtained from Taipei Medical University (TMU) Hospital, Shuang Ho Hospital and the TMU Joint Biobank. Three sets of tissue microarrays of breast cancer tissues were performed to analyze TMEM240 protein expression. Two sets of microarrays were performed in the Department of Pathology of Shuang Ho Hospital. The tissue microarrays contained breast tumor tissues and matched adjacent normal breast tissues obtained from 36 Taiwanese breast cancer cases. Three tissue microarrays representing a total of 131 tissues, including 76 breast carcinoma tissues, 10 matched metastatic carcinoma tissues and 45 matched normal tissues from South Korea, were purchased from SuperBioChips Laboratories (catalog number CBA4; South Korea), and tissues from Taiwanese breast cancer cases were obtained from the Department of Pathology, Shuang Ho Hospital (Taiwan). The pathologic diagnoses of these cases were microscopically confirmed by two researchers. Prior to the collection of clinical data and samples, written informed consent was obtained from all patients. Patients undergoing preoperative chemoradiotherapy or an emergent operative procedure were excluded from this study. Sections of cancerous tissue and corresponding noncancerous tissues were reviewed by a senior pathologist. Clinical data regarding age, sex, tumor type, TNM tumor stage, menopausal state, estrogen receptor (ER), progesterone receptor (PR), and human epidermal growth factor receptor 2 (HER2) tumor markers, were prospectively collected and obtained from Taipei Medical University (TMU) Hospital, Shuang Ho Hospital and the TMU Joint Biobank. Following surgery, patients were monitored every 3 months for the first 2 years and semiannually thereafter.

\section{Genomic DNA, circulating cell-free DNA and RNA extraction}

Genomic DNA from matched pairs of primary tumors and adjacent breast tissues was extracted using the QIAamp DNA Mini Kit (Qiagen, Bonn, Germany, Cat. No. 51306) according to the manufacturer's instructions. The tumor and normal specimens that were used for RNA extraction were frozen immediately after surgical resection and stored at $-80^{\circ} \mathrm{C}$. Total mRNA was extracted using the RNeasy Plus Mini Kit (Qiagen, Hilden, Germany, Cat. No. 74134) according to the manufacturer's instructions. Circulating cell-free DNA was extracted from plasma (3.5 $\mathrm{mL})$ that had been isolated from $10 \mathrm{~mL}$ of peripheral blood within 2 hours of collection. Circulating cell-free DNA (ccfDNA) was extracted from 15 of the plasma samples using the MagMAX Cell-Free DNA Isolation Kit (Thermo Fisher Scientific, Austin, TX, USA) according to the manufacturer's recommended protocol [22-24]. CcfDNA was extracted from 46 of the plasma samples using the iCatcher Circulating cfDNA 1000 kit (CatchGene, New Taipei City, Taiwan) according to the manufacturer's recommended protocol.

\section{Reverse transcription PCR}

To measure TMEM240 mRNA expression, real-time reverse transcription PCR (RT-PCR) was performed in a LightCycler 96 (Roche Applied Science, Penzberg, Germany). Real-time PCR was performed using the SensiFAST ${ }^{\mathrm{TM}}$ Probe No-ROX Kit (Bioline, London, UK, Cat. No. BIO-86020) with specific primers and the corresponding Universal Probe Library probe (Roche Applied Science, Mannheim, Germany) according to the manufacturer's instructions. The glyceraldehyde 3-phosphate dehydrogenase gene (GAPDH) was used as a reference gene. The PCR conditions were as follows: preincubation at $95^{\circ} \mathrm{C}$ for 10 minutes followed by 40 cycles of amplification at $95^{\circ} \mathrm{C}$ for 10 seconds and $60{ }^{\circ} \mathrm{C}$ for 10 seconds. The normalized gene expression values obtained using LightCycler Relative Quantification software (Version 1.5, Roche Applied Science) were compared with those of the control group. TMEM240 mRNA expression was considered low if the mRNA expression level of TMEM240 relative to GAPDH was 0.5-fold lower in the breast tumor tissue than in the paired normal breast tissue. The primers and probes used in RT-PCR are listed in Table S1.

\section{TaqMan quantitative methylation-specific PCR}

After bisulfite conversion of DNA using the EpiTect Fast DNA Bisulfite Kit (Qiagen, Bonn, Germany, Cat. No. 59826), the DNA methylation level of TMEM240 was measured using TaqMan quantitative methylation-specific PCR (QMSP) in a LightCycler 96 (Roche Applied Science, Penzberg, Germany). QMSP was performed using the SensiFAST ${ }^{\mathrm{TM}}$ Probe No-ROX Kit (Bioline, London, UK, Cat. No. BIO-86020) with specific primers and methyl-TaqMan probe for TMEM240. Normalized DNA methylation values, which were calibrated to the control group, were obtained using LightCycler Relative Quantification software (Version 1.5, Roche Applied Science). The beta-actin (ACTB) gene was used as a reference gene. Primers and probes for TMEM240 methylation detection were designed to bind to the junction between the promoter and exon 1. The QMSP conditions were as follows: preincubation at $95^{\circ} \mathrm{C}$ for 10 minutes followed by 50 cycles of amplification at $95^{\circ} \mathrm{C}$ for 10 seconds and $60{ }^{\circ} \mathrm{C}$ for 10 seconds. TMEM240 was considered hypermethylated when the methylation level of TMEM240 relative to that of the ACTB gene was at least 2 -fold higher in the breast tumor than in the paired normal breast tissue sample. The specificity of TMEM240 methylation end products was confirmed by bisulfite sequencing (Figure S1). The primers and probes used in QMSP are listed in Supplementary Table S1.

\section{Genome-wide methylation analysis}


Genome-wide methylation analysis of 5 paired breast cancer tissues and corresponding noncancerous breast tissues was performed using the Illumina Infinium HumanMethylation450 BeadChip array (Illumina, San Diego, CA, USA) for one sample and the Infinium MethylationEPIC Kit (IIlumina) for the remaining 4 samples, as previously reported [17]. The two arrays contain more than 450,000 and 850,000 methylation sites, respectively, and provide genome-wide coverage of the gene region and CpG island coverage, respectively, including $99 \%$ of RefSeq genes. Bisulfite conversion of $500 \mathrm{ng}$ of genomic DNA was performed using the EpiTect Fast DNA Bisulfite Kit (Qiagen, Bonn, Germany, Cat. No. 59826). Methylation scores for each CpG site were represented as "beta" values ranging from 0 (unmethylated) to 1 (fully methylated) based on determination of the ratios of the methylated signal intensities to the sums of the methylated and unmethylated signal outputs.

\section{Cell lines, cell culture, and drug treatment}

The MDA-MB-231 and T47D breast cancer cell lines used in this study were obtained from the Bioresource Collection and Research Center (http://www.bcrc.firdi.org.tw/). MDA-MB-231 cells were cultured in DMEM/F12 supplemented with human platelet lysate (hPL, American Red Cross, USA) and 1\% penicillin/streptomycin. T47D cells were cultured in DMEM/F12 supplemented with human platelet lysate (hPL, American Red Cross, USA), $1 \%$ penicillin/streptomycin and $6 \mathrm{ng} / \mathrm{ml}$ insulin. For the TMEM240 demethylation assay, MDA-MB-231 cells were treated with dimethyl sulfoxide (DMSO) or with the demethylation agent decitabine (DAC, Sigma-Aldrich, St. Louis, MO, USA) for 96 hours. DAC was dissolved in DMSO. After treatment of the cells, DNA and RNA were extracted, and methylation and gene expression levels were analyzed. For the hormone therapy response assay, T47D cells were treated with DMSO or with a series of concentrations of Tamoxifen ( 0,10 and $20 \mu \mathrm{M})$ for 48 hours (Sigma-Aldrich, St. Louis, MO, USA).

\section{Immunofluorescence assay}

For immunofluorescence staining assays, cells were seeded in 4-well glass chamber slides (Nunc). After TMEM240 plasmid overexpression, the DLD-1 cells were fixed in 4\% formaldehyde and stained with anti-DDK (1:200, Abcam, Cambridge, UK). Imaging was performed using deconvolution fluorescence microscopy (Olympus).

\section{Immunohistochemical assay}

Immunohistochemical staining with an antibody against TMEM240 (1:35, Sigma-Aldrich, HPA066721, St. Louis, MO, USA) was performed using an iView DAB detection kit (Ventana, Tucson, AZ, USA) and a BenchMark XT autostainer. The assay included both positive and negative controls. The researchers who evaluated the immunohistochemical staining results were blinded to the clinical follow-up data. The intensity of TMEM240 expression was identified semiquantitatively as no expression, low expression (weaker than or equal to the expression intensity observed in normal colon epithelium), or high expression (stronger than the expression intensity observed in normal colon epithelium).

\section{Plasmid extraction, confirmation and purification}

Plasmid DNA was extracted using the Geneaid ${ }^{\text {TM }}$ Midi Plasmid Kit (Geneaid Biotech Ltd., Cat. No. PI025) according to the manufacturer's instructions. The extracted DNA was subjected to preliminary length analysis by sequenced to confirm errorless production. The plasmid concentration was measured using a NanoDrop 2000C ultramicrowavelength spectrometer (Thermo Fisher Scientific, USA), and the plasmid was stored at $-20^{\circ} \mathrm{C}$ until further use.

\section{cDNA expression construct, RNAi, and transfection}

TMEM240 interference RNA was obtained from Life Technologies Corporation. Transfections were performed using 10 nM si-TMEM240 or nontargeting siRNAs, and Lipofectamine-RNAiMax and Lipofectamine 3000 reagent (Invitrogen) was used to transfect MDA-MB-231 and T47D cells according to the manufacturer's protocol.

\section{Transwell assay}

Transwell assays were used to study cell migration. In the transwell assays, the upper and lower chambers of the culture wells were separated by a semipermeable membrane (Falcon) with a pore size of $8 \mu \mathrm{m}$. Approximately $2 \times 10^{4}$ and $1 \times 10^{5}$ treated and untreated MDA-MB-231 and T47D cells, respectively, were seeded in the upper chamber. Then, $300 \mu \mathrm{L}$ of serum-free DMEM/F12 was added as culture medium, and $800 \mu \mathrm{L}$ of serumcontaining culture medium was added as a chemical attractant in the lower chamber. After 16 hours of incubation, the cells retained over the membrane were washed twice with PBS, fixed with $4 \%$ formaldehyde and stained with $1 \%$ crystal violet/dd $\mathrm{H}_{2} \mathrm{O}$ for 60 minutes at room temperature. Five randomly chosen areas were photographed using a camera attached to a microscope (Nikon), and ImageJ was used to quantify the number of cells in each area.

\section{Wound healing assay}

The wound healing assays were performed using culture inserts (Ibidi, GmbH, Martinsried, Germany). After seeding $1 \times 10^{5}$ cells overnight, the cells were transfected with siRNA for 48 hours. The culture inserts were then removed, the wounded areas were photographed using a camera attached to a microscope (Nikon), and ImageJ was used to calculate the wound areas. 
A sulforhodamine B (SRB) assay was used to measure the cell proliferation rate. MDA-MB-231 and T47D cells were seeded in 96-well plates at densities of $8 \times 10^{3}$ and $1 \times 10^{4}$ cells/well, respectively, and incubated for $24 \mathrm{~h}$ and $48 \mathrm{~h}$, respectively. The cells were then fixed with $10 \%$ trichloroacetic acid for $10 \mathrm{~min}$. After staining with SRB for $30 \mathrm{~min}$, excess dye was removed by washing the cells five times with $1 \%$ acetic acid. Cell proliferation was assessed using a microplate reader to determine the absorbance of the SRB solution at $515 \mathrm{~nm}$.

\section{Statistical analysis}

All statistical analyses were performed using SPSS (SPSS Inc., Chicago, IL, USA). Pearson's chi-squared test was used to compare breast cancer patients with respect to TMEM240 methylation, RNA expression, and clinical data including age, sex, tumor type, TNM tumor stage, race, menopausal state, and ER, PR and HER2 status. The t-test was used to compare cells transfected with or without TMEM240 plasmid or si-TMEM240 and cells with and without drug treatment. Multivariate Cox proportional hazards regression analyses (adjusted for age, sex, race, tumor subtype, and tumor stage) were further used to analyze the correlation between TMEM240 hypermethylation and 10-year overall survival in breast cancer patients. Comparisons of hypermethylation and hypomethylation curves that yielded log-rank test $p$ values of less than 0.05 were considered statistically significant. The TMEM240 methylation level and drug treatment response in breast cancer patients whose data were accessed through the TCGA portal were analyzed using the Mann-Whitney test. In addition to accuracy, other commonly used measures of evaluating the classification, such as the receiver operating characteristic curve (ROC) and area under the curve (AUC), sensitivity, specificity, false-positive rate and false-negative rate, are also reported.

\section{Results}

\section{TMEM240 was identified in samples from Taiwanese and Western breast cancer patients by genome-wide methylation analysis}

To identify a novel potential biomarker in breast cancer patients with poor hormone therapy response, we used five criteria to screen potential targets: (1) hypermethylation in Taiwanese breast cancer patients; (2) hypermethylation in Western breast cancer patients; (3) hypermethylation in breast cancer patients with poor hormone therapy response compared with complete response; (4) a methylation level in normal tissues that was close to 0; and (5) low expression in breast cancer patients (Figure 1A). First, to identify critical tumor suppressor genes, the Infinium Methylation Assay was applied to 5 breast cancer tissue samples and paired noncancerous breast tissue samples. A total of 2612 genes were hypermethylated according to the criterion $\triangle$ Avg_ $\beta$ ( $\beta$ Tumor $-\beta$ Normal) $>0.4$. Second, we analyzed the TCGA Illumina Infinium HumanMethylation450 BeadChip array data of 87 paired Western breast cancer patients. A total of 6882 genes were found to be hypermethylated according to the criterion $\Delta$ Avg_ $\beta$ $(\beta$ Tumor $-\beta$ Normal) $>0.4$. Next, the top 20 genes with the highest methylation levels in 8 breast cancer patients with poor hormone therapy response compared with 21 patients with complete response to hormone therapy were identified. Next, we further found that 11940 genes showed much lower DNA methylation levels in breast, colon, rectal, lung, uterine, gastric, esophageal, pancreatic, liver, and prostate normal tissues. Finally, TCGA RNA sequencing data of 38 paired breast cancer samples from Western patients showed that the expression of 2474 genes were decreased by $50 \%$ in the breast cancer samples. The TMEM240 gene was identified using InteractiVenn (Figure 1B). Few reports about TMEM240 in women cancer was found. Methylation of TMEM240 was further analyzed in the TCGA cohort, and the gene was found to be highly methylated in breast cancer, endometrial and uterine cancer. The cluster analysis of the TMEM240 methylation pattern was visualized as a heatmap (Figure 1C). The role of TMEM240 in breast cancer is unclear. Therefore, TMEM240 in breast cancer was selected for further analysis. A comprehensive analysis of its epigenetic alterations, mRNA and protein expression was performed, and the biological role of TMEM240 was further studied.

\section{TMEM240 represses breast cancer cell proliferation and cell migratory ability}

Alterations in TMEM240 and its functional roles during tumorigenesis have not been studied previously. To study the biological roles of the TMEM240 protein in breast cancer cells, TMEM240 was overexpressed or knocked down in MDA-MB-231 cells by electroporation. The gene manipulation efficiency was determined through real-time RT-PCR. Transfection of the TMEM240 plasmid into MDA-MB-231 and T47D cells resulted in abundant TMEM240 protein expression (Figure 2A, left panel) and mRNA expression (Figure 2A-B, middle panel). According to the SRB cell viability assay, TMEM240 inhibited MDA-MB-231 and T47D cancer cell growth by $55.2 \%$ and $48.7 \%$, respectively (Figure $2 A-B$, right panel). Microscopic observation revealed that TMEM240 overexpression repressed the growth of T47D and MDA-MB-231 cells compared with a vector control (Figure 2B, left panel; and Figure S2). To determine whether decreased TMEM240 expression induces cell growth, TMEM240 gene expression was knocked down in MDA-MB-231 and T47D cells using si-TMEM240. TMEM240 mRNA expression was reduced after transfection of the MDA-MB231 and T47D cells with si-TMEM240 for $24 \mathrm{~h}$ compared with the ci-control (Figure 2C and 2D). Microscopic observation and SRB assay revealed that si-TMEM240 induced proliferation of MDA-MB-231 and T47D cells compared with the si-control group. TMEM240 knockdown increased MDAMB-231 and T47D cell proliferation by 1.4- and 1.5-fold, respectively (Figure 2C and 2D).

To investigate whether TMEM240 is associated with breast cancer cell migration, MDA-MB-231 cells were transfected with TMEM240 or siTMEM240 for $24 \mathrm{~h}$. The motility of the cells was then analyzed using transwell assays and wound healing assays. The data revealed that an increase in TMEM240 expression suppressed the migration ability of MDA-MB-231 cells by 75.6\% (Figure 2E). Knockdown of TMEM-240 in MDA- 
MB-231 cells significantly induced cell migration by $63.0 \%$ and $62.7 \%$ based on the results obtained using transwell assays (Figure $2 \mathrm{~F}$ ) and wound healing assays (Figure $2 \mathrm{G}$ ), respectively.

\section{TMEM240 protein is mainly distributed in cell membranes and in the cytoplasm}

Although TMEM240 is predicted to be a membrane protein, to date no study has reported the intracellular distribution of the TMEM240 protein. According to the structure of TMEM240 protein reported in the UniProtKB/Swiss-Prot database, the protein contains two transmembrane protein regions located between amino acid residues 5 25 and 90 110. Exogenous expression of TMEM240 and immunofluorescent staining were used to examine the distribution of the protein in the DLD-1 cell line. Deconvolution and 3D reconstruction of immunofluorescence images showed that the TMEM240 protein was mainly concentrated in the cytoplasm and cell membranes (Figure 4).

\section{Low TMEM240 protein expression in Taiwanese and South Korean breast cancer patients}

TMEM240 reveals the tumor suppressor potential for breast cancer cell growth and migration (Figure 2). To investigate whether TMEM240 protein expression is altered in cancerous breast tissues, TMEM240 protein expression in 76 breast tumors from 40 Korean breast cancer patients and 36 Taiwanese breast cancer patients was analyzed by immunohistochemistry. TMEM240 was observed to be localized to the cell membrane and cytoplasm in normal breast tissues (Figure 3A). The protein was expressed at lower-than-normal levels in 88.2\% (67/76) of the tumors from breast cancer patients and in $90.0 \%$ (9/10) of metastatic tumors from breast cancer patients (Figure 3B and 3C and Table 1). Almost all triple-negative breast cancer patients $(95.7 \%, 22 / 23)$ had deficient TMEM240 protein expression (Table 1$)$.

\section{Promoter hypermethylation and low TMEM240 mRNA expression in Taiwanese breast cancer patients}

Low expression of TMEM240 protein was observed in breast cancer patients. We investigated whether TMEM240 mRNA was also expressed at lower levels in breast cancer. We analyzed TMEM240 mRNA expression in 52 paired Taiwanese breast cancer tissues. In 50.0\% (26/52) of these tissues, TMEM240 mRNA expression was lower in the breast cancer tumor tissue than in the normal breast tissue (Figure 3D, Table 1). We further analyzed the methylation patterns of TMEM240 in paired 101 Taiwan breast cancer patients, the methylation level of TMEM240 was at least 2-fold higher in 54.5\% (55/101) breast tumor tissues than in the matched normal breast tissues (Figure 3E, 3F and Table 1). The DNA hypermethylation levels and mRNA expression levels of TMEM240 showed a significant negative correlation by Spearman rank correlation coefficient analysis ( $p=$ 0.037). To determine whether hypermethylation of TMEM240 is involve in the regulation of mRNA expression, TMEM240 mRNA expression was investigated using administration of the DNA demethylating drug decitabine (DAC) to T47D and MDA-MB-231 breast cancer cells. The cells were treated with DMSO and DAC for $48 \mathrm{~h}$. In the DAC groups for the two cell lines, methylation of TMEM240 decreased to $28.36 \%$ and $7.8 \%$, respectively, of that in the DMSO group ( $p=0.001$, Figure $3 \mathrm{G}$ and $3 \mathrm{H}$, left panel), and TMEM240 mRNA expression increased by 150-fold and 337-fold, respectively $(p<0.001$, Figure $3 \mathrm{G}$ and $3 \mathrm{H}$, right panel), suggesting that hypermethylation of the TMEM240 promoter is the main mechanism through which TMEM240 silencing occurs.

\section{TMEM240 promoter hypermethylation and low mRNA expression in breast cancer tissues from the TCGA dataset}

To further evaluate alterations in TMEM240 hypermethylation and mRNA expression in Western breast cancer patients, we analyzed the TCGA data of the Illumina Infinium HumanMethylation450 BeadChip array for 78 breast cancer tumors, 78 matched normal tissues and 623 breast cancer tumor tissues and displayed the methylation levels in a heatmap. The exon 1 region of TMEM240 was hypermethylated in $40.3 \%$ (251/623) of the breast tumor tissues (Table 2). Analysis of RNA sequencing data from TCGA showed that TMEM240 mRNA expression was reduced by half in $51.4 \%(37 / 72)$ of the breast cancer tumor tissues compared with the matched normal breast tissues $(p=0.019$, Figure S3) and in $60.2 \%(458 / 761)$ of tumors from breast cancer patients (Table 2). The DNA hypermethylation levels and mRNA expression levels of TMEM240 showed a significant negative correlation on Spearman rank correlation coefficient analysis ( $p=0.049)$. Hypermethylation of TMEM240 was associated with Asian, ERnegative, PR-negative and triple-negative breast cancer patients and patients with invasive ductal carcinoma (all $p<0.001$, Table 2). In addition, Kaplan-Meier curves indicated that patients with hypermethylation of TMEM240 had a poor survival rate (Figure 4A, log rank test, $p=0.003$ ). A Cox proportional hazards survival analysis further adjusted for race, age, tumor type, tumor stage and menopausal state showed that $T M E M 240$ promoter hypermethylation was significantly and independently associated with 10-year overall survival $($ Table $3, p=0.002)$.

\section{Hypermethylation of TMEM240 in breast cancer was associated with poor treatment response in the TCGA cohort set}

To further investigate whether hypermethylation of $T M E M 240$ is associated with poor treatment response, we analyzed the relationship between hypermethylation of TMEM240 and clinical treatment response to chemotherapy, hormone therapy and targeted therapy in patients from the TCGA cohort. The results indicated that patients with hypermethylation of TMEM240 had poor chemotherapy response (Table 4, $p=0.012)$ and poor hormone therapy response (Table $4, p<0.001$ ). Better hormone therapy response was observed in $85.0 \%$ of patients with lower methylation of TMEM240 but in only $28.6 \%$ of patients with hypermethylation of TMEM240 (Table 4B, Mann-Whitney U test, $p=0.005$ ). Higher methylation of TMEM240 was associated with poorer response to tamoxifen treatment (Figure 4C, Mann-Whitney U test, $p=0.041$ ) and with poorer response to aromatase inhibitor treatment (Figure 4D, Mann-Whitney U test, $p=0.037$ ).

High TMEM240 expression enhance the tamoxifen treatment response in breast cancer cell lines 
To further investigate whether the expression of TMEM240 may be involved in the response to hormone drug treatment, a cell proliferation assay was performed after overexpression and/or knockdown of TMEM240 and tamoxifen treatment in T47D breast cancer cells. The proliferation of T47D (ER+/PR+) cells was significantly decreased by $62.9 \%$ when cells transfected with si-control were treated with $20 \mathrm{mM}$ tamoxifen $(p=0.003)$, but only a $31.1 \%$ decrease in proliferation was observed in cancer cells transfected with si-TMEM240 (Figure 4E). The data indicate that lower expression of TMEM240 is related to resistance to tamoxifen treatment. Overexpression of TMEM240 in T47D cells induced 76.9\% cancer cell death when the cells were treated with $20 \mathrm{mM}$ tamoxifen but only a $46.0 \%$ decrease in the vector control cancer cells when treated with 20 mM tamoxifen (Figure 4F).

\section{Circulating methylated TMEM240 predicts disease progression and poor hormone therapy response in Taiwanese breast cancer patients}

Hypermethylation of TMEM240 was found in breast tumors of patients who displayed poor treatment response, especially in tumors from patients who received hormone therapy. Detection of circulating methylated TMEM240 in the plasma of patients with poor treatment response could provide a potential tool for real-time monitoring of clinical outcomes after medical treatment. Breast cancer patients were recruited from the Taipei Medical University Hospital and Shuang Ho Hospital and were followed up for at least 1 year. After these patients received treatment, circulating methylated DNA was extracted from their plasma at 3-6 month intervals and analyzed by QMSP. The patients with poor prognosis had significantly higher circulating methylated TMEM240 levels than other patients but did not display higher levels of CA-15-3 and CEA (Table 5). The level of circulating methylated TMEM240 dramatically and gradually decreased in breast cancer patients following treatment (Case 1 and Case 2, Figure 5A-B). When patients experienced disease progression, recurrence or metastasis, the levels of circulating methylated TMEM240 increased significantly (Case 3 and Case 4, Figure 5C-E, Mann-Whitney U test, $p<0.001$ ). The circulating methylated TMEM240 test for poor prognosis prediction was found to have $87.5 \%$ sensitivity $(28 / 32), 93.1 \%$ specificity $(27 / 29)$, and $90.2 \%$ accuracy $(55 / 61)$, values that are better than those obtained using the currently used biomarkers CEA and CA-153 (Table 5 and Figure 5).

Hypermethylation of TMEM240 in breast cancer was associated with poor response to hormone therapy in the TCGA cohort. We further investigated whether patients with an increase in circulating methylated TMEM240 in plasma experienced disease progression, recurrence or metastasis after hormone therapy. The results indicated that the patients with poor response after hormone therapy had significantly higher levels of circulating methylated TMEM240 (Table 5). The circulating methylated TMEM240 test for poor hormone therapy response prediction was found to have a sensitivity of $76.9 \%(10 / 13)$, a specificity of $95.7 \%(22 / 23)$, and an accuracy of $88.9 \%(32 / 36)$ (Figure $5 E-F)$.

\section{Discussion}

Aberrant promoter hypermethylation of $\mathrm{CpG}$ islands associated with TSGs can cause transcriptional silencing and contribute to tumorigenesis. In the present investigation, hypermethylation of TMEM240 in patients with poor hormone therapy response was identified using genome-wide methylation array analysis. QMSP confirmed the presence of TMEM240 hypermethylation in Taiwanese breast cancer tumor tissues compared with normal tissues. In the TCGA cohort, hypermethylation of the promoter region of TMEM 240 was found in $40.3 \%$ of tumors. It occurs more frequently in Asian patients (54.8\%). Results similar to those found in the Asian TCGA cohort were consistently found in Taiwanese breast cancer patients (54.5\%). Low expression of TMEM240 protein was found in most Taiwanese and Korean breast cancer patients. Moreover, patients with hypermethylation of TMEM240 had poor 10-year overall survival. In addition, hypermethylation of TMEM240 was observed in patients with progressive disease, especially in patients treated with hormone therapy. The results obtained for Taiwanese breast cancer patients were similar to those obtained for patients in the TCGA cohort. Hypermethylation of TMEM240 in $87.8 \%$ of CRC, $80.0 \%$ of esophageal cancer and $80.4 \%$ of liver cancer patients was reported in our previous study [13]. Here, we further analyzed a TCGA cohort and found high methylation of TMEM240 in breast cancer and in endometrial and uterine cancer. Alterations in TMEM240 in female cancer are less frequent than those that occur in gastrointestinal cancer, but they are associated with poor clinical treatment response and poor prognosis in breast cancer. Circulating methylated TMEM240 dramatically and gradually decreased and then diminished in breast cancer patients without disease progression, whereas it increased in breast cancer patients with recurrence or metastasis. Hypermethylation of TMEM240 leads to low expression of TMEM240 mRNA and low TMEM240 protein expression. TMEM240 expression induces breast cancer cell death and enhances the cellular response to hormone therapy drugs, suggesting that deficiency in TMEM240 expression plays an important role during cancer progression in breast cancer patients.

In addition to its association with progressive disease and poor prognosis, hypermethylated TMEM240 was found to be strongly associated with ER/PR negative breast cancer, TNBC and poor hormone therapy response. Almost all triple-negative breast cancer patients (95.7\%, 22/23) displayed deficient TMEM240 protein expression. Patients with hypermethylation of TMEM240 often had poor hormone therapy response (Table $3, p<$ 0.001). Patients who had circulating hypermethylated TMEM240 also experienced disease progression (Figure 7). The data indicate that TMEM240 deficiency is involved in breast tumorigenesis through pathways other than the ER/PR and HER2 pathways, leading to a poor hormone therapy response. Even patients with hypermethylation of TMEM240 and positive ER/PR expression exhibited poor hormone therapy responses, including treatment with tamoxifen or aromatase inhibitors (Als) (Table 4). Only in patients in which TMEM240 was expressed at sufficient levels in the cancer cells did hormone therapy produce a good therapeutic response (Figure 2 and Figure 3 ). $\mathrm{Ki}-67$ as a parameter and multigene analysis (MGA) have been used to predict the response to hormone therapy [25, 26]. Methylation of TMEM240 may play a role in determining resistance to Al drugs such as Ki-67 or MGA. The mechanisms and pathways that are modulated by TMEM240 are worth investigating further. TNBC represents a group of breast cancers with heterogeneous genomic features. There are several different subtypes of

Page $7 / 21$ 
TNBC, including the Vanderbilt subtype and the Baylor subtype [27, 28]. Each subtype carries a different set of mutant genes [27, 28]. Further study may focus on the relationship between TMEM240 and specific subtypes of TNBC.

Advances in detection technology have reduced breast cancer death rates in several Western countries [15]. Therefore, the development and use of biomarkers of treatment response can improve patient outcomes. The presence of breast-derived circulating DNA is indicative of residual disease after treatment [29]. Circulating methylated TMEM240 dramatically and gradually decreases and then diminishes in patients with various subtypes of breast cancer who do not show disease progression (Figure 7), suggesting that measurement of circulating methylated TMEM240 could be used to detect the presence of residual disease. In addition, the level of circulating methylated TMEM240 in plasma increased further in breast cancer patients with recurrence or metastasis (Figure 7). In these patients, the concentrations of CEA and CA15-3 in serum remained normal or increased much later than did the circulating methylated TMEM240. The detection of CEA and CA15-3 was incapable of revealing disease progression and poor treatment response in several patients (Table 5). Measurement of circulating methylated TMEM240 could be used to monitor and detect early disease progression after treatment and during long-term follow-up. Although hypermethylation of $T M E M 240$ also occurs in other types of cancer, its high alteration in cancers may assist the detection of disease progression. Combining measurement of TMEM240 hypermethylation with the measurement of additional breast cancer-specific methylated DNA biomarkers that are associated with disease progression will improve detection sensitivity and cancer specificity.

\section{Conclusion}

Deficiency in TMEM240 expression plays an important role during cancer progression in breast cancer patients. Circulating hypermethylated TMEM240 may represent a potential biomarker for disease progression and poor hormone therapy response.

\section{Abbreviations}

ACTB, beta-actin; CA15-3, cancer antigen 15-3;ccfDNA, circulating cell-free DNA; CEA, carcinoembryonic antigen; cmDNA, circulating methylated DNA; breast cancer, breast cancer; GAPDH, glyceraldehyde 3-phosphate dehydrogenase gene; Ki-67, marker of proliferation Ki-67; QMSP, quantitative methylation specific real-time polymerase chain reaction; QPCR, Quantitative real-time reverse transcription polymerase chain reaction; SAC21, spinocerebellar ataxia 21; SRB, sulforhodamine B; TNBC, Triple negative breast cancer; TCGA, The Cancer Genome Atlas; TMEM240, transmembrane Protein 240; TSG, tumor suppressor gene; TMU, Taipei Medical University; QS, overall survival.

\section{Declarations}

\section{Ethics approval and consent to participate}

The study has been approved by the Taipei Medical University - Joint Institutional Review Board and the Institutional Review Board. Written informed consent was obtained from all patients.

\section{Consent for publication}

Not applicable

\section{Availability of data and materials}

The data generated in this study are available from the corresponding author upon reasonable request.

\section{Competing interests}

There are no conflicts of interest.

\section{Funding}

This work was supported in part by MOST108-2320-B-038-020 and 110-2321-B-038 -003 from the Ministry of Science and Technology (Republic of China, Taiwan); 110-5804-001-400 from the Ministry of Education (Republic of China, Taiwan).

\section{Authors' contributions}

CSH and RKL designed the studies.

$\mathrm{CSH}, \mathrm{CMS}$ and HET collected and provided the clinical samples.

SYL, LTAT, PLL and JYC performed the experiments.

$\mathrm{RKL}, \mathrm{CMS}$ and $\mathrm{CSH}$ analyzed and interpreted the data. 
RKL and CSH drafted the manuscript.

All authors read and approved the manuscript.

Acknowledgments: We thank the Core Facility Center of Taipei Medical University for providing the LightCycler 480 system (Roche Applied Science), FACSCanto II flow cytometer (BD Biosciences), Deconvolution Fluorescence Microscope (Olympus), and Olympus IX71 Inverted Microscope System.

\section{Authors' information}

${ }^{1}$ Division of Colon and Rectal Surgery, Department of Surgery, Taipei Veterans General Hospital, Taipei, Taiwan. ${ }^{2}$ Department of Pathology, Shuang Ho Hospital, Taipei Medical University, New Taipei, Taiwan. ${ }^{3}$ Department of Pathology, School of Medicine, College of Medicine, Taipei Medical University, Taipei, Taiwan. ${ }^{4}$ Ph.D. Program for the Clinical Drug Discovery from Botanical Herbs, Taipei Medical University, Taipei, Taiwan.

${ }^{5}$ Department of Surgery, School of Medicine, College of Medicine, Taipei Medical University, Taipei, Taiwan. ${ }^{6}$ Division of General Surgery, Department of Surgery, Shuang Ho Hospital, Taipei Medical University, New Taipei City, Taiwan. ${ }^{7}$ Ph.D. Program in Biotechnology Research and Development, College of Pharmacy, Taipei Medical University, Taipei, Taiwan. ${ }^{8}$ School of Pharmacy, College of Pharmacy, Taipei Medical University, Taipei, Taiwan. ${ }^{9}$ Graduate Institute of Pharmacognosy, Taipei Medical University, Taipei, Taiwan. ${ }^{10}$ Master Program for Clinical Pharmacogenomics and Pharmacoproteomics, Taipei, Taiwan. ${ }^{11}$ Clinical trial center, Taipei Medical University Hospital, Taipei, Taiwan, R.O.C.

\section{References}

1. Cancer Registry Annual Report, 2017, Taiwan, Health Promotion Administration Ministry of Health and Welfare, Taiwan. 2019.

2. Ministry of Health and Welfare, Taiwan, R.O.C. 2020. Available at https://www.mohw.gov.tw/cp-16-48057-1.html.

3. Bray F, Ferlay J, Soerjomataram I, Siegel RL, Torre LA, Jemal A. Global cancer statistics 2018: GLOBOCAN estimates of incidence and mortality worldwide for 36 cancers in 185 countries. CA Cancer J Clin. 2018; 68(6):394-424.

4. Zhu W, Xu B. Overcoming resistance to endocrine therapy in hormone receptor-positive human epidermal growth factor receptor 2-negative $(\mathrm{HR}(+) / \mathrm{HER} 2(-))$ advanced breast cancer: a meta-analysis and systemic review of randomized clinical trials. Frontiers of medicine. 2021; 15(2):208-20.

5. Ma CX, Bose R, Ellis MJ. Prognostic and Predictive Biomarkers of Endocrine Responsiveness for Estrogen Receptor Positive Breast Cancer. Advances in experimental medicine and biology. 2016; 882:125-54.

6. Sharma S, Kelly TK, Jones PA. Epigenetics in cancer. Carcinogenesis. 2010; 31(1):27-36.

7. Costello JF, Fruhwald MC, Smiraglia DJ, Rush LJ, Robertson GP, Gao X, Wright FA, Feramisco JD, Peltomaki P, Lang JC et al. Aberrant CpG-island methylation has non-random and tumour-type-specific patterns. Nat Genet. 2000; 24(2):132-8.

8. Delplanque J, Devos D, Huin V, Genet A, Sand O, Moreau C, Goizet C, Charles P, Anheim M, Monin ML et al. TMEM240 mutations cause spinocerebellar ataxia 21 with mental retardation and severe cognitive impairment. Brain : a journal of neurology. 2014; 137(Pt 10):2657-63.

9. Traschutz A, van Gaalen J, Oosterloo M, Vreeburg M, Kamsteeg EJ, Deininger N, Riess O, Reimold M, Haack T, Schols L et al. The movement disorder spectrum of SCA21 (ATX-TMEM240): 3 novel families and systematic review of the literature. Parkinsonism \& related disorders. 2019; 62:215-20.

10. Yahikozawa H, Miyatake S, Sakai T, Uehara T, Yamada M, Hanyu N, Futatsugi Y, Doi H, Koyano S, Tanaka F et al. A Japanese Family of Spinocerebellar Ataxia Type 21: Clinical and Neuropathological Studies. Cerebellum (London, England). 2018; 17(5):525-30.

11. Zeng S, Zeng J, He M, Zeng X, Zhou Y, Liu Z, Xia K, Pan Q, Jiang H, Shen L et al. Spinocerebellar ataxia type 21 exists in the Chinese Han population. Scientific reports. 2016; 6:19897.

12. Seki T, Sato M, Kibe Y, Ohta T, Oshima M, Konno A, Hirai H, Kurauchi Y, Hisatsune A, Katsuki H. Lysosomal dysfunction and early glial activation are involved in the pathogenesis of spinocerebellar ataxia type 21 caused by mutant transmembrane protein 240 . Neurobiology of disease. $2018 ; 120: 34-50$

13. Chang SC, Liew PL, Ansar M, Lin SY, Wang SC, Hung CS, Chen JY, Jain S, Lin RK. Hypermethylation and decreased expression of TMEM240 are potential early-onset biomarkers for colorectal cancer detection, poor prognosis, and early recurrence prediction. Clin Epigenetics. 2020; 12(1):67.

14. Naumov VA, Generozov EV, Zaharjevskaya NB, Matushkina DS, Larin AK, Chernyshov SV, Alekseev MV, Shelygin YA, Govorun VM. Genome-scale analysis of DNA methylation in colorectal cancer using Infinium HumanMethylation450 BeadChips. Epigenetics. 2013; 8(9):921-34.

15. DeSantis CE, Ma J, Gaudet MM, Newman LA, Miller KD, Goding Sauer A, Jemal A, Siegel RL. Breast cancer statistics, 2019. CA Cancer J Clin. 2019; 69(6):438-51.

16. Dawson SJ, Tsui DWY, Murtaza M, Biggs H, Rueda OM, Chin SF, Dunning MJ, Gale D, Forshew T, Mahler-Araujo B et al. Analysis of Circulating Tumor DNA to Monitor Metastatic Breast Cancer. New Engl J Med. 2013; 368(13):1199-209.

17. Duffy MJ, Evoy D, McDermott EW. CA 15-3: uses and limitation as a biomarker for breast cancer. Clinica chimica acta; international journal of clinical chemistry. 2010; 411(23-24):1869-74.

Page $9 / 21$ 
18. Banin Hirata BK, Oda JM, Losi Guembarovski R, Ariza CB, de Oliveira CE, Watanabe MA. Molecular markers for breast cancer: prediction on tumor behavior. Disease markers. 2014; 2014:513158.

19. Li Z, Guo X, Tang L, Peng L, Chen M, Luo X, Wang S, Xiao Z, Deng Z, Dai L et al. Methylation analysis of plasma cell-free DNA for breast cancer early detection using bisulfite next-generation sequencing. Tumour Biol. 2016; 37(10):13111-19.

20. Wan JCM, Massie C, Garcia-Corbacho J, Mouliere F, Brenton JD, Caldas C, Pacey S, Baird R, Rosenfeld N. Liquid biopsies come of age: towards implementation of circulating tumour DNA. Nat Rev Cancer. 2017; 17(4):223-38.

21. Amatu A, Schirripa M, Tosi F, Lonardi S, Bencardino K, Bonazzina E, Palmeri L, Patane DA, Pizzutilo EG, Mussolin B et al. High Circulating Methylated DNA Is a Negative Predictive and Prognostic Marker in Metastatic Colorectal Cancer Patients Treated With Regorafenib. Frontiers in oncology. 2019; 9:622.

22. Yan L, Chen Y, Zhou J, Zhao H, Zhang H, Wang G. Diagnostic value of circulating cell-free DNA levels for hepatocellular carcinoma. International journal of infectious diseases : IJID : official publication of the International Society for Infectious Diseases. 2018; 67:92-97.

23. Hung CS, Wang SC, Yen YT, Lee TH, Wen WC, Lin RK. Hypermethylation of CCND2 in Lung and Breast Cancer Is a Potential Biomarker and Drug Target. International journal of molecular sciences. 2018; 19(10).

24. Wang SC, Liao LM, Ansar M, Lin SY, Hsu WW, Su CM, Chung YM, Liu CC, Hung CS, Lin RK. Automatic Detection of the Circulating Cell-Free Methylated DNA Pattern of GCM2, ITPRIPL1 and CCDC181 for Detection of Early Breast Cancer and Surgical Treatment Response. Cancers. $2021 ; 13(6)$.

25. Untch M, Thomssen C, Bauerfeind I, Braun M, Brucker SY, Felberbaum R, Hagemann F, Haidinger R, Hönig A, Huober J et al. Primary Therapy of Early Breast Cancer: Evidence, Controversies, Consensus: Spectrum of Opinion of German Specialists on the 16th St. Gallen International Breast Cancer Conference (Vienna 2019). Geburtshilfe und Frauenheilkunde. 2019; 79(6):591-604.

26. Yerushalmi R, Woods R, Ravdin PM, Hayes MM, Gelmon KA. Ki67 in breast cancer: prognostic and predictive potential. The Lancet Oncology. 2010; 11(2):174-83.

27. Lehmann BD, Bauer JA, Chen X, Sanders ME, Chakravarthy AB, Shyr Y, Pietenpol JA. Identification of human triple-negative breast cancer subtypes and preclinical models for selection of targeted therapies. J Clin Invest. 2011; 121(7):2750-67.

28. Ahn SG, Kim SJ, Kim C, Jeong J. Molecular Classification of Triple-Negative Breast Cancer. Journal of breast cancer. 2016; 19(3):223-30.

29. Moss J, Zick A, Grinshpun A, Carmon E, Maoz M, Ochana BL, Abraham O, Arieli O, Germansky L, Meir K et al. Circulating breast-derived DNA allows universal detection and monitoring of localized breast cancer. Annals of oncology : official journal of the European Society for Medical Oncology. 2020; 31(3):395-403.

\section{Tables}

Table 1. TMEM240 protein, mRNA expression and promoter hypermethylation in relation to the clinical parameters of Taiwan breast cancer. ${ }^{1}$ 


\begin{tabular}{|c|c|c|c|c|c|c|}
\hline \multirow[t]{2}{*}{ Characteristics } & \multirow[t]{2}{*}{$A \|^{2}$} & Protein expression & \multirow[t]{2}{*}{ All } & mRNA expression ${ }^{3}$ & \multirow[t]{2}{*}{ All } & DNA Methylation ${ }^{4}$ \\
\hline & & Low n(\%) High n(\%) & & Low n(\%) High n(\%) & & Low n(\%) High n(\%) \\
\hline
\end{tabular}

Overall

$\begin{array}{lllll}76 & 67 & (88.2) & 9 & (11.8)\end{array}$

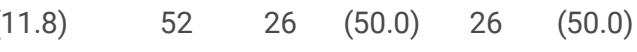

$101 \quad 46 \quad(8.9)$

(54.5)

Tumor Type

$\begin{array}{llllllllllllllll}\text { IDC } & 60 & 52 & (86.7) & 8 & (13.3) & 52 & 26 & (50.0) & 26 & (50.0) & 98 & 43 & (43.9) & 55 & (56.1) \\ \text { ILC } & 1 & 1 & (100.0) & 0 & (0.0) & 0 & 0 & (0.0) & 0 & (0.0) & 2 & 2 & (100.0) & 0 & (0.0) \\ \text { Others } & 5 & 5 & (100.0) & 0 & (0.0) & 0 & 0 & (0.0) & 0 & (0.0) & 1 & 1 & (100.0) & 0 & (0.0)\end{array}$

Tumor Stage

\begin{tabular}{|c|c|c|c|c|c|c|c|c|c|c|c|c|c|c|c|}
\hline $0, I$ and II & 48 & 41 & (85.4) & 7 & (14.6) & 9 & 5 & (55.6) & 4 & (44.4) & 19 & 7 & (36.8) & 12 & $(63.2)$ \\
\hline III and IV & 18 & 17 & (94.4) & 1 & (5.6) & 41 & 21 & (51.2) & 20 & (48.8) & 79 & 38 & (48.1) & 41 & (51.9) \\
\hline
\end{tabular}

Tumor Size

\begin{tabular}{llllllllllllllll} 
T0-T1 & 11 & 10 & $(90.9)$ & 1 & $(9.1)$ & 40 & 24 & $(60.0)$ & 16 & $(40.0)^{0.040}$ & 30 & 12 & $(40.0)$ & 28 & $(60.0)$ \\
\hline T2-T4 & 52 & 46 & $(88.5)$ & 6 & $(11.5)$ & 9 & 2 & $(22.2)$ & 7 & $(77.8)$ & 68 & 33 & $(48.5)$ & 35 & $(51.5)$
\end{tabular}

Lymph node regional metastasis

\begin{tabular}{llllllllllllllll} 
No & 27 & 25 & $(92.6)$ & 2 & $(7.4)$ & 23 & 14 & $(60.9)$ & 9 & $(39.1)$ & 43 & 21 & $(48.8)$ & 22 & $(51.2)$ \\
\hline Yes & 36 & 31 & $(86.1)$ & 5 & $(13.9)$ & 25 & 11 & $(44.0)$ & 14 & $(56.0)$ & 51 & 24 & $(47.1)$ & 27 & $(52.9)$
\end{tabular}

Grade

\begin{tabular}{llllllllllllllll} 
Well & 4 & 3 & $(75.0)$ & 1 & $(25.0)$ & 7 & 3 & $(42.9)$ & 4 & $(57.1)^{7}$ & 10 & 5 & $(50.0)$ & 5 & $(50.0)$ \\
\hline Moderate & 27 & 22 & $(81.5)$ & 5 & $(18.5)$ & 17 & 9 & $(52.9)$ & 8 & $(47.1)$ & 40 & 17 & $(42.5)$ & 23 & $(57.5)$ \\
\hline Poor & 29 & 27 & $(93.1)$ & 2 & $(6.9)$ & 24 & 13 & $(54.2)$ & 11 & $(45.8)$ & 42 & 20 & $(47.6)$ & 22 & $(52.4)$
\end{tabular}

ER

$\begin{array}{llllllllllllllll}\text { Negative } & 39 & 35 & (89.7) & 4 & (10.0) & 15 & 8 & (53.3) & 7 & (46.7) & 31 & 14 & (45.2) & 17 & (54.8) \\ \text { Positive } & 37 & 32 & (86.5) & 5 & (13.5) & 27 & 13 & (48.1) & 14 & (51.9) & 68 & 30 & (44.1) & 38 & (55.9)\end{array}$

PR

$\begin{array}{llllllllllllllll}\text { Negative } & 42 & 39 & (92.9) & 3 & (7.1) & 16 & 9 & (56.3) & 7 & (43.8) & 40 & 16 & (40.0) & 24 & (60.0) \\ \text { Positive } & 34 & 28 & (82.4) & 6 & (17.6) & 26 & 12 & (46.2) & 14 & (53.8) & 59 & 28 & (47.5) & 31 & (52.5)\end{array}$

HER2

$\begin{array}{llllllllllllllll}\text { Negative } & 42 & 51 & (92.7) & 4 & (7.3)^{0.046} & 18 & 10 & (55.6) & 8 & (44.4) & 39 & 18 & (46.2) & 21 & (53.8) \\ \text { Positive } & 21 & 16 & (76.2) & 5 & (23.8) & 22 & 20 & (45.5) & 3 & (54.5) & 59 & 25 & (42.4) & 34 & (57.6)\end{array}$

TNBC

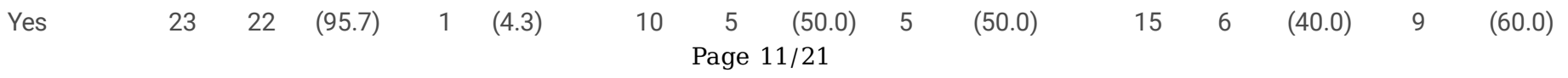




\section{Ki-67}

\begin{tabular}{llllllllllllllll}
$<14 \%$ & 10 & 9 & $(90.0)$ & 1 & $(10.0)$ & 11 & 5 & $(45.5)$ & 6 & $(54.5)$ & 26 & 13 & $(50.0)$ & 13 & $(50.0)$ \\
$\geqq 14 \%$ & 16 & 11 & $(68.8)$ & 5 & $(31.3)$ & 23 & 10 & $(43.5)$ & 13 & $(56.5)$ & 66 & 27 & $(40.9)$ & 39 & $(59.1)$ \\
\hline
\end{tabular}

p53

$\begin{array}{llllll}\text { Negative } & 26 & 26 & (100.0) & 0 & (0.0)\end{array}$

$\begin{array}{lllll}\text { Positive } & 22 & (88.0) & 3 & (12.0)\end{array}$

1. These results were analyzed by the Pearson $X^{2}$ test. $P$ values with significance are shown as superscripts.

2. For some categories, the number of samples (n) was lower than the overall number analyzed because clinical data were unavailable for those samples.

3. When the TMEM240 expression level in breast tumors was less than half of the mean of TMEM240 expression levels in adjacent normal breast tissues was defined as low expression.

4. The TMEM240 promoter methylation level in breast tumors being 2-fold higher than in adjacent normal breast tissues was defined as hypermethylation.

Table 2. TMEM240 mRNA expression and promoter hypermethylation in relation to the clinical parameters of breast cancer from TCGA. ${ }^{1}$ 
Characteristics

Total
Low High
$n(\%) \quad n(\%)$
Low High
$n(\%) \quad n(\%)$

TMEM240 Methylation

Overall

\begin{abstract}
714
\end{abstract}
$427 \quad(59.8)$

287

(40.2)

$582 \quad 351$

(60.3)

231

(39.7)

\section{Age \\ $\geq 65$}

$<65$

$$
210
$$

Race

White

Black/African

American

Asian

ran

525

$421 \quad 259$

$72 \quad 41$

$\begin{array}{llll}32 & 27 & (84.4) & 5\end{array}$

\begin{tabular}{lll}
$(38.5)$ & 338 & 234 \\
\hline$(43.1)$ & 64 & 19 \\
\hline$(15.6)^{0.022}$ & 31 & 14
\end{tabular}

$\begin{array}{llll}(84.4) & 5 & (15.6)^{0.022} & 31\end{array}$

Menopause State $\quad 480$

\begin{tabular}{llllllllllll} 
Premenopause & 123 & 76 & $(61.8)$ & 47 & $(38.2)$ & 103 & 69 & $(67.0)$ & 34 & $(33.0)$ \\
Perimenopause & 16 & 9 & $(56.3)$ & 7 & $(43.8)$ & 14 & 6 & $(42.9)$ & 8 & $(57.1)$ \\
\hline Postmenopause & 341 & 218 & $(63.9)$ & 123 & $(36.1)$ & 287 & 174 & $(60.6)$ & 113 & $(39.4)$
\end{tabular}

Histological Type $\quad 714$

\begin{tabular}{lcccccccccc} 
ILC & 170 & 65 & $(38.2)$ & 105 & $(61.8)$ & 152 & 113 & $(74.3)$ & 39 & $(25.7)$ \\
\hline IDC & 504 & 341 & $(67.7)$ & 163 & $(32.3)^{<0.001}$ & 400 & 215 & $(58.8)$ & 185 & $(46.3)^{<0.001}$ \\
$\begin{array}{l}\text { Mucinous } \\
\text { Carcinoma }\end{array}$ & 15 & 8 & $(53.3)$ & 7 & $(46.7)$ & 14 & 11 & $(78.6)$ & 3 & $(21.4)$ \\
Mixed type & 25 & 13 & $(52.0)$ & 12 & $(48.0)$ & 16 & 12 & $(75.0)$ & 4 & $(25.0)$
\end{tabular}

Tumor Stage $\quad 535$

$\begin{array}{lllllllllll}\text { I and II } & 385 & 247 & (64.2) & 138 & (35.8) & 311 & 189 & (60.8) & 122 & (39.2) \\ \text { III and IV } & 150 & 86 & (57.3) & 64 & (42.7) & 129 & 80 & (62.0) & 49 & (38.0)\end{array}$

Tumor Size $\quad 539$

$\begin{array}{lllllllllll}\text { T0-T1 } & 137 & 93 & (67.9) & 44 & (32.1) & 114 & 80 & (70.2) & 34 & (29.8) \\ \text { T2-T4 } & 402 & 243 & (60.4) & 159 & (39.6) & 329 & 191 & (58.1) & 138 & (41.9)^{0.022}\end{array}$

ER
\begin{tabular}{|lllllllllll} 
\\
Negative & 535 & & & & & & & \\
& 117 & 84 & $(71.8)$ & 33 & $(28.2)^{0.023}$ & 103 & 22 & $(21.4)$ & 81 & $(78.6)^{<0.001}$ \\
Positive & 418 & 252 & $(60.3)$ & 166 & $(39.7)$ & 340 & 249 & $(73.2)$ & 91 & $(26.8)$
\end{tabular}




\begin{tabular}{|c|c|c|c|c|c|c|c|c|c|c|}
\hline PR & 533 & & & & & & & & & \\
\hline Negative & 169 & 118 & (69.8) & 51 & $(30.2)^{0.020}$ & 143 & 45 & (31.5) & 98 & $(68.5)^{<0.001}$ \\
\hline Positive & 364 & 216 & (59.3) & 148 & (40.7) & 299 & 225 & (75.3) & 74 & $(24.7)$ \\
\hline HER2 & 463 & & & & & & & & & \\
\hline Negative & 404 & 245 & $(60.6)$ & 159 & (39.4) & 340 & 220 & $(64.7)$ & 120 & (35.3) \\
\hline Positive & 59 & 41 & (69.5) & 18 & (30.5) & 45 & 20 & (44.4) & 25 & $(55.6)^{0.008}$ \\
\hline TNBC & 515 & & & & & & & & & \\
\hline Yes & 76 & 54 & (71.1) & 22 & (28.9) & 68 & 13 & (19.1) & 55 & $(80.9)^{<0.001}$ \\
\hline No & 439 & 267 & $(60.8)$ & 172 & (39.2) & 358 & 255 & (71.2) & 103 & $(28.8)$ \\
\hline
\end{tabular}

1. These results were analyzed by the Pearson $X^{2}$ test. $P$ values with significance are shown as superscripts.

2. When the TMEM240 expression level in breast tumors was less than half of the mean of TMEM240 expression levels in adjacent normal breast tissues was defined as low expression from TCGA data set using RNA sequencing analysis.

Table 3 Cox proportional hazard model of clinical parameters and TMEM240 DNA methylation level associated with breast cancer

\begin{tabular}{|c|c|c|c|c|c|c|}
\hline \multirow{3}{*}{$\begin{array}{l}\text { Breast } \\
\text { Cancer } \\
\text { Variable }\end{array}$} & \multicolumn{6}{|c|}{ 10-year overall survival ${ }^{1}$} \\
\hline & \multicolumn{3}{|c|}{ Univariate analysis } & \multicolumn{3}{|c|}{ Multivariate analysis } \\
\hline & $\mathrm{HR}$ & $95 \% \mathrm{Cl}$ & $P$-value & HR & $95 \% \mathrm{Cl}$ & $P$-value \\
\hline Race & 0.902 & $0.568-1.433$ & 0.662 & 1.389 & $0.553-3.489$ & 0.484 \\
\hline Age & 2.055 & $1.366-3.091$ & $0.001 * * *$ & 1.516 & $0.508-4.529$ & 0.456 \\
\hline Tumor type & 1.391 & $1.028-1.882$ & $0.033^{*}$ & 1.241 & $0.567-2.716$ & 0.588 \\
\hline Stage & 2.052 & $1.311-3.213$ & $0.002^{\star \star}$ & 4.098 & $1.551-10.827$ & $0.004 * \star \star$ \\
\hline Menopause & 1.256 & $0.904-1.746$ & 0.174 & 1.498 & $0.787-2.815$ & 0.221 \\
\hline TMEM240 & 2.747 & $1.364-5.531$ & $0.005^{\star \star}$ & 6.172 & 1.984-19.197 & $0.002^{\star \star}$ \\
\hline
\end{tabular}

1. These results were analyzed by the Cox regression model.

2. The TMEM240 DNA methylation levels were derived from 640 breast cancer patients in TCGA data set.

3. ${ }^{*} P<0.05 ; * \star, P<0.01 ; * \star *, P<0.001$.

Table 4. TMEM240 promoter hypermethylation in relation to drug treatment response in TCGA cohort. ${ }^{1}$ 


\begin{tabular}{|c|c|c|c|c|}
\hline \multirow[t]{3}{*}{ Characteristics } & \multirow[t]{3}{*}{ Total } & Complete & Progressive & \multirow{3}{*}{$P$ value } \\
\hline & & response & disease & \\
\hline & & $\mathbf{N}(\%)$ & $\mathbf{N}(\%)$ & \\
\hline Chemotherapy? & 257 & $239(93.0)$ & $18(7.0)$ & \\
\hline Low methylation & 99 & $97(98.0)$ & $2(2.0)$ & 0.012 \\
\hline High methylation & 158 & $142(89.9)$ & $16(10.1)$ & \\
\hline
\end{tabular}

\section{Hormone therapy $\underline{\underline{3}}$}

$\begin{array}{llll}\text { Low methylation } & 24 & 21(87.5) & 3(12.5) \\ \text { High methylation } & 11 & 2(18.2) & 9(81.8)\end{array}$

\section{Targeted Molecular therapy 4}

\begin{tabular}{cccc} 
Low methylation & 4 & $4(100.0)$ & $0(0.0)$ \\
\hline High methylation & 8 & $6(75.0)$ & $2(25.0)$
\end{tabular}

1. These results were analyzed by the Fisher's exact test. The patients with a treatment duration of greater than 4 weeks were included in this analysis. When the $\beta$ value of TMEM240 methylation level in breast tumors was higher than 0.25 was defined as hypermethylation from TCGA data set using Infinium Human Methylation 450K BeadChip.

2. Chemotherapy drugs:

antimetabolites drugs: 5-fluorouracil, capecitabine, gemcitabine, methotrexate.

alkylating drugs: cyclophosphamide, cisplatin and carboplatin.

topoisomerase inhibitors: doxorubicin, mitoxantrone and epirubicin.

microtubule inhibitors: taxanes, vinca alkaloids, and epothilones.

1. Hormone therapy drug:

Estrogen inhibitors: tamoxifen and fulvestrant.

aromatase inhibitors: letrozole, anastrozole and exemestane.

1. Targeted Molecular therapy: Avastin and Herceptin.

Table 5. TMEM240 promoter hypermethylation in relation to prognosis and drug treatment response in plasma of Taiwanese breast cancer patients. ${ }^{1}$ 


\begin{tabular}{|c|c|c|c|c|}
\hline \multirow[t]{3}{*}{ Characteristics } & \multirow[t]{3}{*}{ Total $^{2}$} & & \multicolumn{2}{|l|}{ Progressive $^{3}$} \\
\hline & & Progression & disease & $P$ value \\
\hline & & $N(\%)$ & $\mathbf{N}(\%)$ & \\
\hline Overall & 61 & $29(47.5)$ & $32(52.5)$ & \\
\hline \multicolumn{5}{|l|}{-} \\
\hline \multicolumn{5}{|l|}{ TMEM240 in plasma } \\
\hline No methylation & 31 & $27(87.1)$ & $4(12.9)$ & \multirow[t]{2}{*}{$<0.001$} \\
\hline High methylation & 30 & $2(6.7)$ & $28(93.3)$ & \\
\hline \multicolumn{5}{|l|}{ CA-153 in serum } \\
\hline Normal & 55 & $29(52.7)$ & $26(47.3)$ & \multirow[t]{2}{*}{0.237} \\
\hline Abnormal (> 25 units $/ \mathrm{ml}$ ) & 2 & 0 (33.3) & $2(100.0)$ & \\
\hline
\end{tabular}

CEA in serum

\begin{tabular}{llcc} 
Normal & 49 & $26(53.1)$ & $23(46.9)$ \\
$\qquad \begin{array}{l}\text { Abnormal }(>5 \mathrm{ng} / \mathrm{ml}) \\
\text { Underwent hormone therapy. }\end{array}$ & 9 & $3(33.3)$ & $6(66.7)$ \\
\hline
\end{tabular}

TMEM240 in plasma

$\begin{array}{llll}\text { Low methylation } & 25 & 22(88.0) & 3(12.0) \\ \text { High methylation } & 11 & 1(9.1) & 10(90.9)\end{array}$

Ki-67 in breast tumors

$\begin{array}{lccc}\text { Low expression } & 16 & 10(62.5) & 6(37.5) \\ \text { High expression }(>15 \%) & 20 & 13(65.0) & 7(35.0)\end{array}$

1. These results were analyzed by the Fisher's exact test. The patients with a treatment and monitoring duration of greater than one year were included in this analysis. When the circulating methylated TMEM240 levels normalized by circulating ACTB in plasma of breast cancer patients was higher than 0.002 was defined as abnormal.

2. For concentration of CA-153, CEA and Ki-67 expression, the number of samples (n) was lower than the overall number analyzed because clinical data were unavailable for those samples.

3. Non-Progression: Patients without Progression, Recurrence, Metastasis.

Progressive disease: Patients with Progression, Recurrence, Metastasis.

\section{Figures}


A

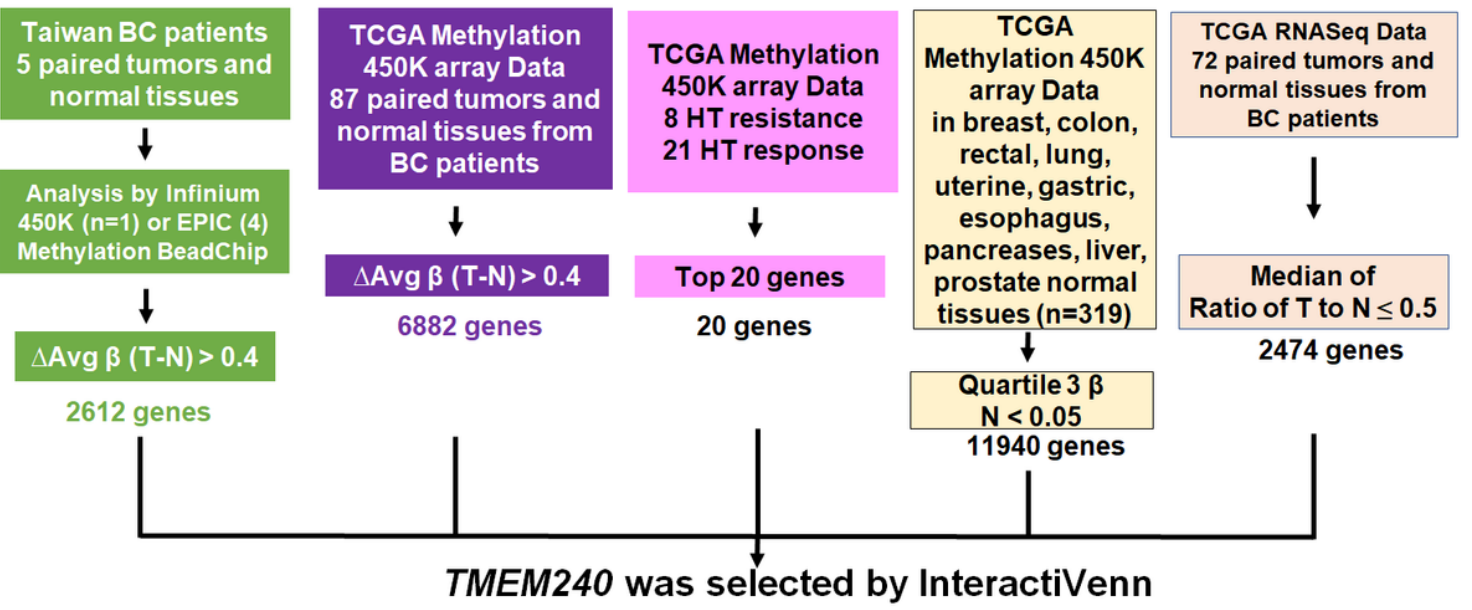

B

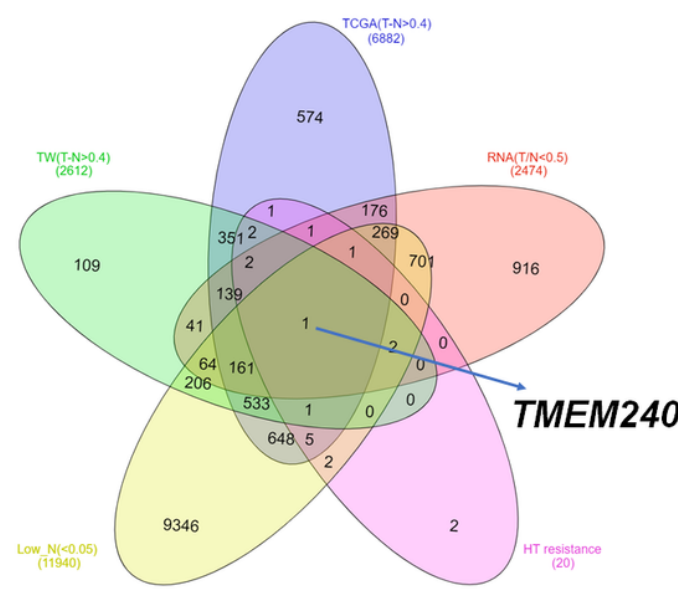

C Methylation level of TMEM240 ( $\beta$ value)

TCGA Breast Cancer TCGA Endometrial Cancer
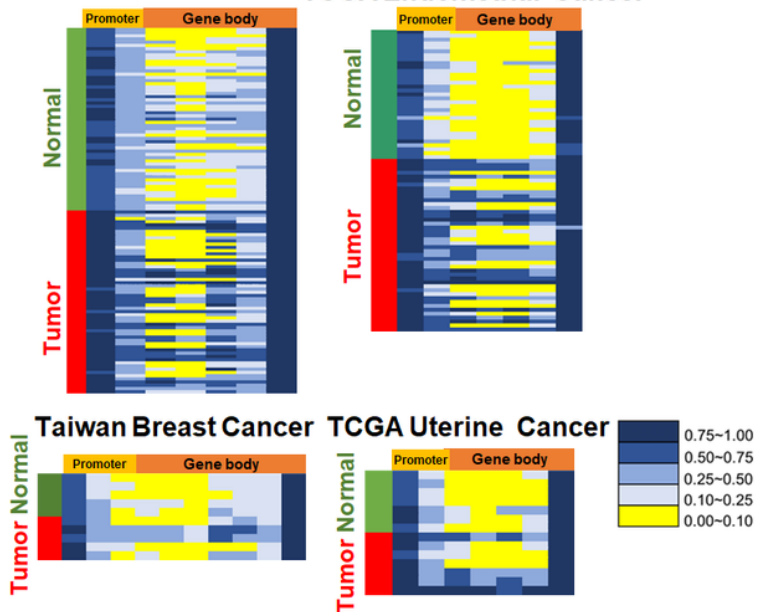

Figure 1

Flowchart of gene selection and heatmap for TMEM240. (A) The criteria and step-by-step flowchart for gene selection. (B) Screening of intersecting genes by InteractiVenn. (C) Heatmap of the TMEM240 methylation pattern in breast cancer and endometrial and uterine cancer. 


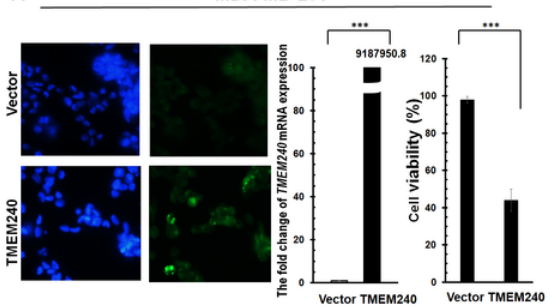

c

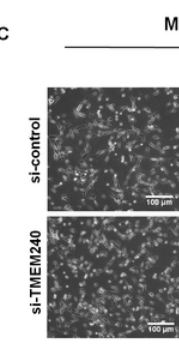

MDA-MB-231
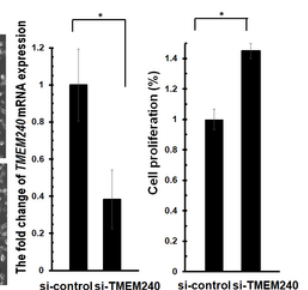

MDA-MB-231
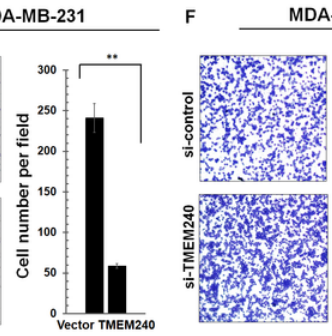

G

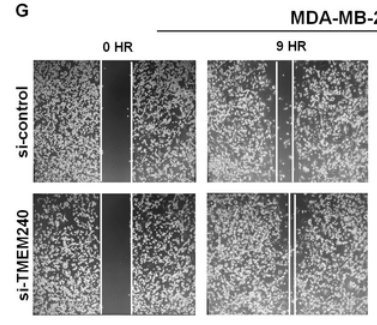

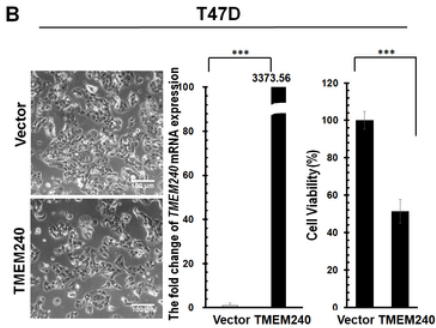

D

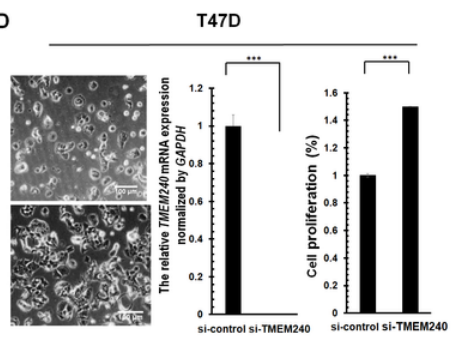

H
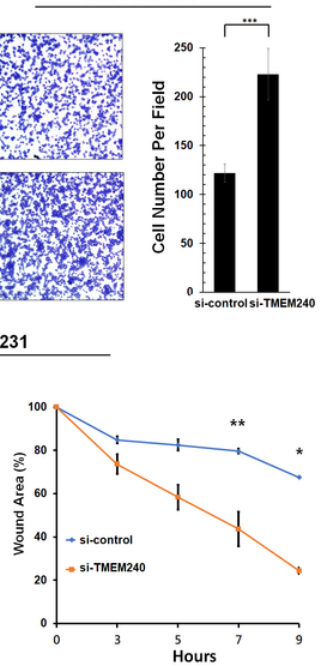

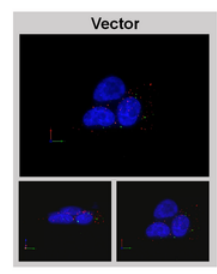

।

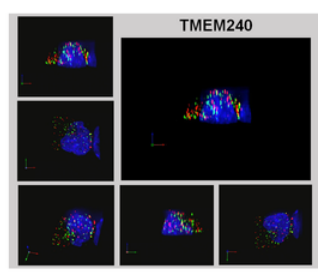

Figure 2

TMEM240 is localized in the cytoplasm and membrane and represses cancer cell growth and migration in breast cancer cells. A recombinant pMycDDK-hTMEM240 plasmid was transfected into MDA-MB-231 breast cancer cells (A) and T47D breast cancer cells (B) for $24 \mathrm{~h}$, and the cells were then analyzed via immunofluorescence for TMEM240 protein (left, original magnification, $\times 200$ ) and real-time RT-PCR for mRNA expression (middle). The proliferation of the MDA-MB-231 and T47D cells was analyzed using sulforhodamine B (SRB) assays (right). si-TMEM240 was transfected into MDA-MB-231 cells (C) and T47D cells (D). The cell morphology (left, original magnification, $\times 100)$, mRNA expression (middle), and rate of cell proliferation (right) of the breast cancer cells were analyzed. (E) The migratory ability of MDA-MB-231 cells after TMEM240 overexpression was measured via transwell assays. si-TMEM240 was transfected into MDA-MB-231 cells for $24 \mathrm{~h}$, and the distribution of the cells was then analyzed using transwell assays $(F)$ and wound healing assays $(G)$. The data are presented as the mean $\pm S D ; * x \leq 0.05,{ }^{* *} p \leq 0.01$, *** $\mathrm{p} \leq 0.001$. A t-test was used to calculate group differences in all experiments. Experiments were performed using at least two biological duplicates and three technical replicates. Localization of the TMEM240 protein was determined by deconvolution and 3D reconstruction. (H) Recombinant pMyc-DDK vector control. (I) The recombinant pMyc-DDK-hTMEM240 plasmid was transfected into the cells for $24 \mathrm{~h}$. Red, anti-Myc-hTMEM240 protein. Green, anti-DDK-hTMEM240 protein. Blue, DAPI staining. 
A Normal mammary gland
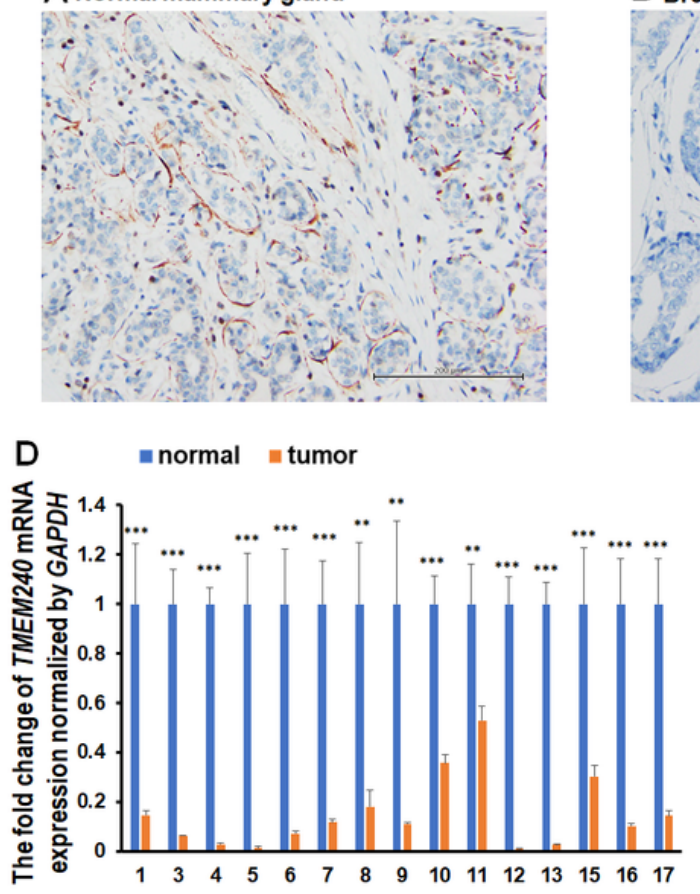

G

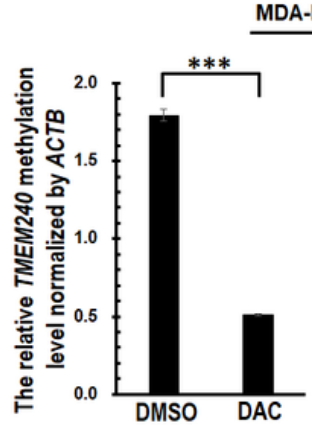

B Breast cancer tissue (negative staining)

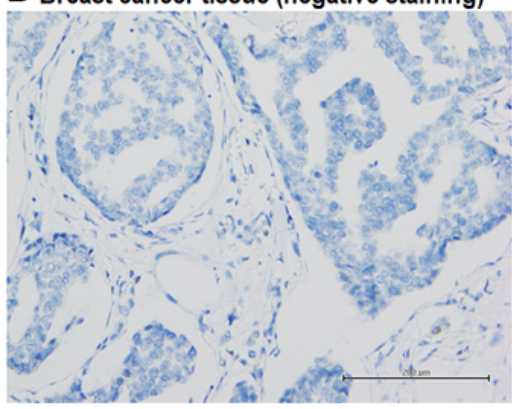

E $\quad$ normal $\approx$ tumor

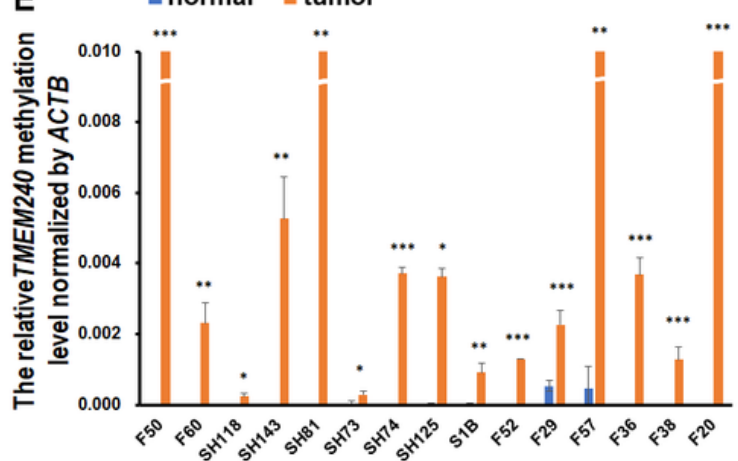

C Breast cancer tissue (positive staining)

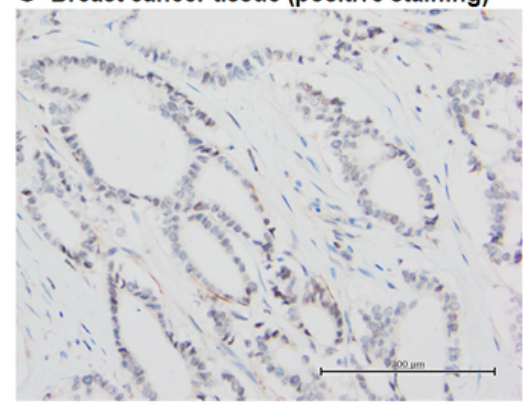

F

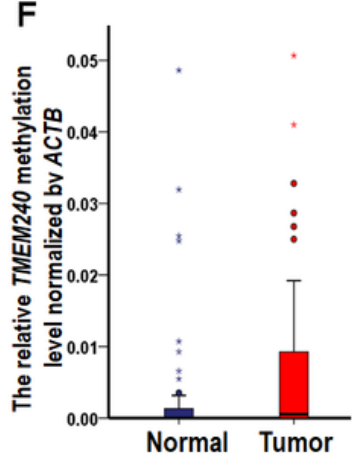

Figure 3

Low expression of TMEM240 in breast cancer is mediated by promoter methylation. Representative figures showing TMEM240 protein expression as analyzed by IHC. (A) Normal mammary gland. (B) Breast cancer tissue with negative expression. (C) Breast cancer tissue with normal expression (original magnification, $\times 200$ ). The scale bars indicate $200 \mu \mathrm{m}$. (D-E) Representative figure showing the TMEM240 mRNA expression level (D) and TMEM240 promoter hypermethylation as determined by RT-qPCR (E) in adjacent breast normal and tumor tissues. (F) Box plot of TMEM240 promoter hypermethylation levels in tissues. $(G, H)$ DNA methylation and mRNA expression were measured after treatment with decitabine (DAC) in MDA-MB-231 (G) and T47D (H) breast cancer cells. The relative DNA methylation levels after treatment with DAC are shown in the left panel. The relative mRNA expression levels after treatment with DAC are shown in the right panel. The data are presented as the mean $\pm S D ; * \star p \leq 0.01, * \star * p$ $\leq$ 0.001. A t-test was used to calculate group differences in all experiments. The experiments were performed using at least two biological duplicates and three technical replicates. 


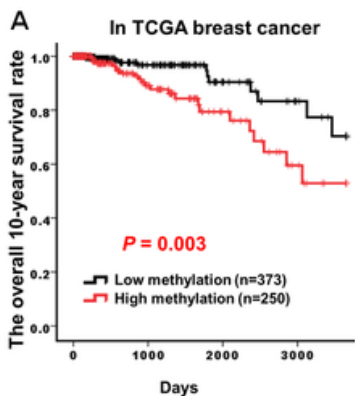

E

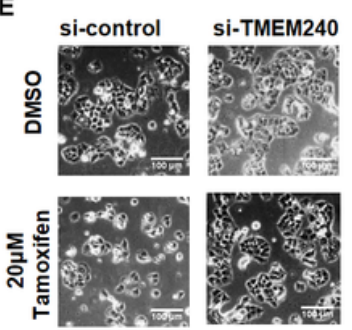

B
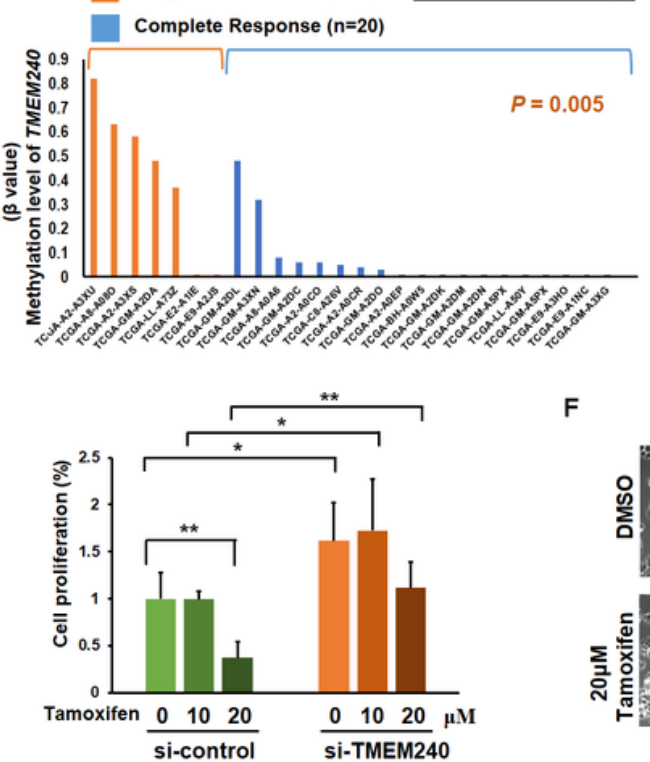

C

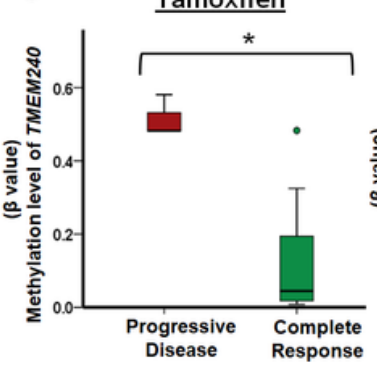

D Aromatase inhibitor

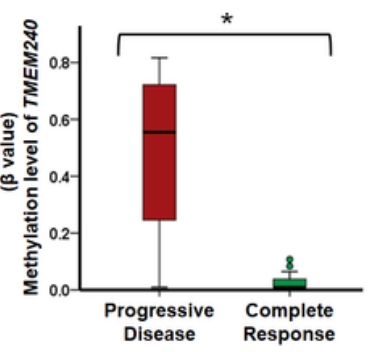

F

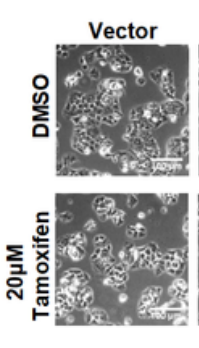

TMEM240

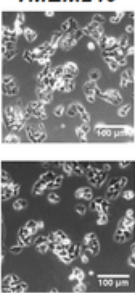

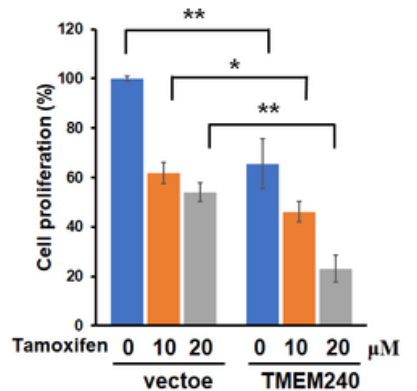

Figure 4

Hypermethylation of TMEM240 is associated with poor hormone therapy response and poor survival in breast cancer. (A) Kaplan-Meier survival curves were used to compare 10-year survival in breast cancer patients with low and high TMEM240 promoter hypermethylation. (B) Bar charts showing TMEM240 methylation levels in breast cancer patients. Orange bar: breast cancer patients with progressive disease after hormone therapy; blue bar, breast cancer patients with complete response after hormone therapy. (C, D) Box plots of TMEM240 methylation levels in patients with complete response or progressive disease after tamoxifen treatment (C) or aromatase inhibitor treatment (D). (E, F) Cell proliferation assays were performed in T47D cells treated with tamoxifen after si-TMEM240 transfection (E, right) or pMyc-DDK-hTMEM240 plasmid transfection (F, right). Brightfield views (E-F, left) are presented to illustrate the cell morphology. 

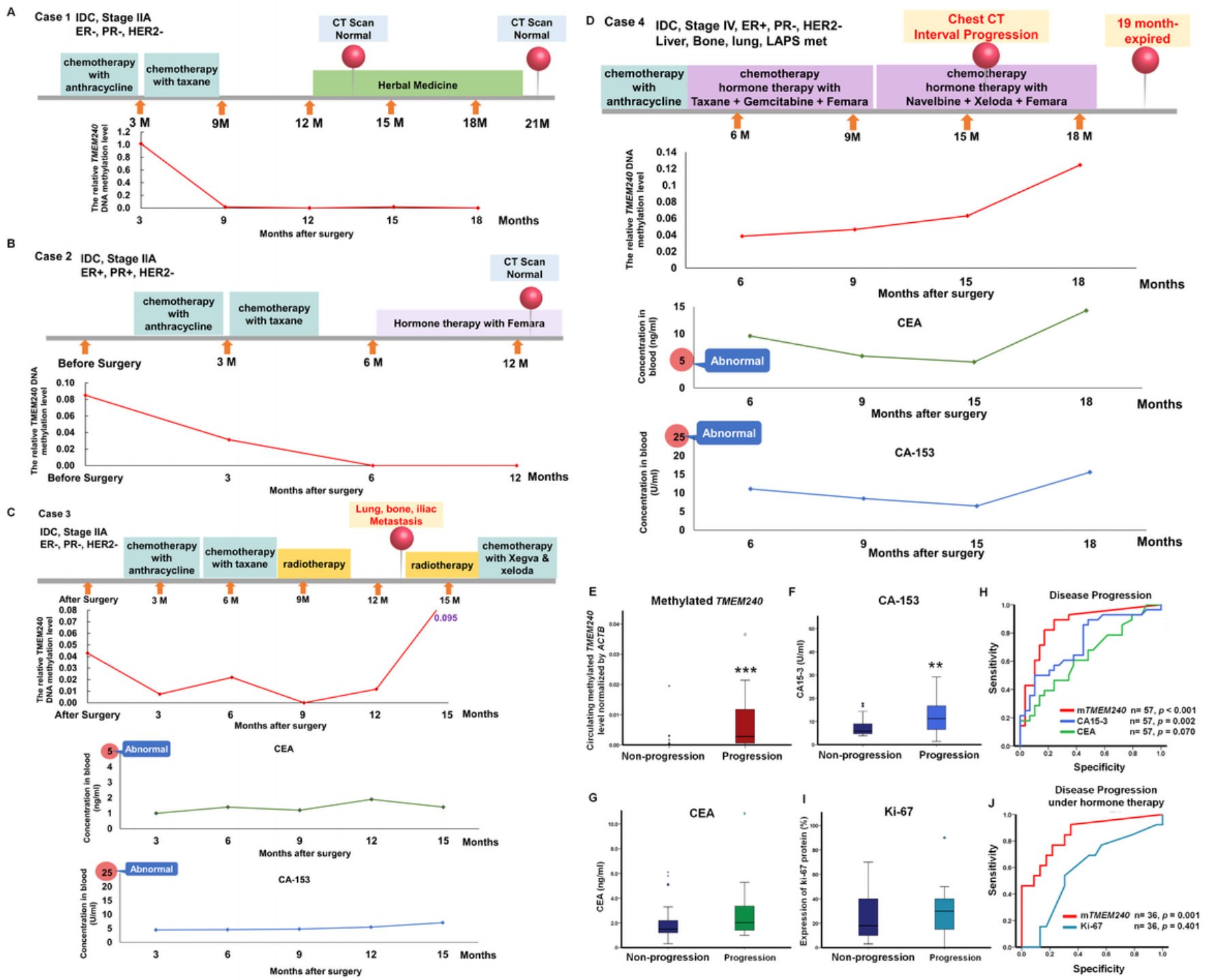

\section{Figure 5}

Circulating methylated TMEM240 is increased in Taiwanese breast cancer patients with disease progression and poor hormone therapy response. The level of circulating methylated TMEM240 dramatically and gradually decreased in triple-negative breast cancer patients (A) and in breast cancer patients who received hormone therapy and did not experience recurrence or metastasis (B). (C) Recurrence and metastasis were detected in triplenegative breast cancer patients when circulating methylated TMEM240 was found in such patients after treatment, and it thereafter increased again. The concentrations of CEA and CA15-3 in serum remained normal. (D) The level of circulating methylated TMEM240 gradually increased in breast cancer patients with disease progression even after hormone therapy and chemotherapy. The concentrations of CEA and CA15-3 in serum are incapable of early monitoring of disease progression and poor treatment response. (E) Box plot showing the levels of circulating methylated TMEM240 in the plasma of 32 patients with recurrence/metastasis and in the plasma of 29 patients without recurrence/metastasis. (F, G) Box plots showing the concentrations of CEA and CA15-3 in the sera of 28 patients with recurrence/metastasis and in the sera of 29 patients without recurrence/metastasis. **, $\mathrm{P}<0.01 ; * \star *, \mathrm{P}<0.001$. $(\mathrm{H})$ ROC curves for disease progression prediction were calculated using the measured circulating methylated TMEM240 levels and the measured concentrations of CEA and CA15-3. (I) A box plot of the percentage of Ki-67 expression level in breast cancer tumors from 13 patients with recurrence/metastasis and in tumors from 23 patients without recurrence/metastasis is shown. (J) ROC curves for disease progression and hormone therapy response prediction were calculated using circulating methylated TMEM240 levels and Ki-67 expression levels.

\section{Supplementary Files}

This is a list of supplementary files associated with this preprint. Click to download.

- Supplementalmeterialsandresults.docx 